The Review of Economic Studies Ltd.

Information and Incentives: The Agency Information Problem

Author(s): Frøystein Gjesdal

Source: The Review of Economic Studies, Vol. 49, No. 3 (Jul., 1982), pp. 373-390

Published by: The Review of Economic Studies Ltd.

Stable URL: http://www.jstor.org/stable/2297362

Accessed: 08/12/2010 08:27

Your use of the JSTOR archive indicates your acceptance of JSTOR's Terms and Conditions of Use, available at http://www.jstor.org/page/info/about/policies/terms.jsp. JSTOR's Terms and Conditions of Use provides, in part, that unless you have obtained prior permission, you may not download an entire issue of a journal or multiple copies of articles, and you may use content in the JSTOR archive only for your personal, non-commercial use.

Please contact the publisher regarding any further use of this work. Publisher contact information may be obtained at http://www.jstor.org/action/showPublisher?publisherCode=resl.

Each copy of any part of a JSTOR transmission must contain the same copyright notice that appears on the screen or printed page of such transmission.

JSTOR is a not-for-profit service that helps scholars, researchers, and students discover, use, and build upon a wide range of content in a trusted digital archive. We use information technology and tools to increase productivity and facilitate new forms of scholarship. For more information about JSTOR, please contact support@jstor.org. 


\title{
Information and Incentives: The Agency Information Problem
}

\author{
FRøYSTEIN GJESDAL \\ Norwegian School of Economics and Business Administration
}

\begin{abstract}
This paper considers the use of imperfect information for risk sharing and incentive purposes when perfect observation of actions and outcomes is impossible, making complete contracting infeasible. The incentive-insurance problem is defined to consist of two parts: the choice of an information system and the design of a sharing rule based on the information system. A generalized agency model is formulated to analyse this problem. The agency models of Ross (1973a, b), Wilson (1968), Stiglitz (1974), Mirrlees (1976), Harris and Raviv (1979), Holmström (1979) a.o. appear as special cases of the generalized model. The analysis focuses on the value of information in the agency information problem. The set of information systems which are valuable-i.e. improve risk sharing and incentives in a Pareto sense-is characterized. A problemindependent ranking of information systems for the agency information problem is then characterized under the assumption that the agent's preferences are additive in money and actions. The ranking may be viewed as a generalization of Blackwell's ranking of information systems for decision problems, to this particular game. When the agent's risk preferences depend on his choice of action, on the other hand, it is shown that the Blackwell ranking may be invalid. Randomized incentive schemes are shown to be efficient when the incentive effect of risk is positive and sufficiently large relative to the absolute risk aversion of the partners.
\end{abstract}

\section{INTRODUCTION}

The principal-agent model is a description of the externality which results when individuals (agents) make decisions without considering how others (principals) are affected by their decisions. ${ }^{1}$ As is well known, there exist incentive mechanisms which, by making the agent's remuneration a function of his actions, will induce Pareto efficient decision making. However, if actions are not costlessly observable, such schemes may be infeasible or suboptimal. This paper considers general two-part incentive mechanisms of the following type. First an information system (control system, performance measurement system) is chosen. Then an incentive scheme is designed with payment to the agent made conditional on the signal received from the information system.

This paper focuses on the first stage-the optimal choice of information system. In Section 3 it will be shown how information systems may be compared for a given agency problem. In particular the set of valuable information systems-those that are strictly preferred to no information-will be characterized. More generally it will be assumed that some information system is costlessly available, and the set of marginally valuable information systems is then characterized. Much of the previous work on agency theory has primarily dealt with the second stage of the incentive mechanism-the design of the incentive scheme for a fixed information system. However, some have explicitly discussed marginal value of information systems, ${ }^{2}$ albeit for special cases, and others may be interpreted as addressing aspects of this issue as well. ${ }^{3}$ Section 3 presents a generalization of this work.

In Section 4 comparisons of mutually exclusive information systems are discussed. A ranking of information systems is characterized which does not depend on the specific agency problem, as long as the agent's utility is additive in money and action. This 
ranking may be viewed as a generalization of Blackwell's ranking of experiments (Blackwell (1951)). The generalized Blackwell ranking may prompt some to suggest that choosing an information system for the agency is formally equivalent to choosing information systems in a Bayesian decision problem if the agent's action is taken to be the unknown state of nature and the incentive scheme to be the decision function. However, the agency is a game, and actions are chosen by a player in the game rather than by nature. Moreover, the Blackwell ranking is only valid for a subclass of agency problems. Leaving this class, randomized transfer payments may very well be efficient. This is conclusive evidence that the decision problem analogy does not work. It is instructive, though, to see how far it goes, and where it breaks down.

The randomization issue is explored in Section 5. The conditions under which the incentive effect of risk is positive, are derived. However, risk itself is negative. Randomization is shown to be efficient only when the net effect is positive. The section is concluded with an analysis of the incentive-risk trade-off under randomization.

In Section 2 the agency model is formulated and discussed. Section 6 contains concluding remarks.

\section{THE MODEL}

$\Theta=\{\theta\}$ and $\mathscr{A}=\left\{a=\left(a_{1}, \ldots, a_{p}\right)\right\}$ are the sets of states and actions respectively. $\Theta$ describes the uncertainty in the economy and $\mathscr{A}$ the decision alternatives. A Borel Field and a probability measure on which the principal and the agent agree, are defined on $\Theta$.

An outcome function is a function $X: \Theta \times \mathscr{A} \rightarrow \mathscr{R}$ where $\mathscr{R}$ is the set of real numbers. $X$ is a random variable on $\Theta$ for each $a .\{x\}$ is the image of $X$, and is assumed to be finite. $x$ may be interpreted as money.

An information system is a function $Y: \Theta \times \mathscr{A} \rightarrow\{y\} . \quad Y$ is a random variable on $\Theta$ for every $a$. The set of signals $\{y\}$ is finite and has cardinality $m$. The notation $Z$ and $\{z\}$ (cardinality $m^{\prime}$ ) will also be used for an information system. If $Z$ and $Y$ are informative systems, the function $(Z, Y)$ whose image is $\{(z, y)\}$, is an information system as well. The signals $y$ and $z$ are observed after the agent has chosen $a$. The set of information systems observed by the principal as well as the agent is denoted $\mathscr{H}$. $\mathscr{H}$ is always assumed to contain at least one constant function which is referred to as no information and denoted $Y^{0}$. A randomization may be defined as an information system which does not depend on $a$, and is statistically independent of $X$. $\mathscr{H}$ may be understood to include randomizations. However, randomizations are a non-issue as long as Assumption 4 below is imposed, as $Y^{0}$ is then preferred to any randomization. $A$ is the information system which is the identity mapping of $\mathscr{A}$ into itself for every $\theta$. When $A \in \mathscr{H}$, the action is said to be observable. $X$ is an information system as well. When $X \in \mathscr{H}$ the outcome is observable.

Information systems which are included in $\mathscr{H}$, are assumed to be costless. On the other hand many information systems which one might want to consider may not be contained in $\mathscr{H}$. The information system $A$ is in general excluded. This is certainly reasonable if $a$ is interpreted as effort. $X$ may not be included either. Many risks are uninsurable because the cost of observing outcomes is larger than the value of insuring. The cost of auditing income tax returns makes for less than perfect audits. If the agent is the manager of a firm, he may want to be paid off before the outcome of his actions is observed (multiperiod considerations such as this one are admittedly ad hoc as only single period models will be considered). Finally, the cost in a cost-plus contract is just an imperfect estimate of the contractor's "real" cost.

When outcome is unobservable, it is important to specify who owns it. ${ }^{4}$ The outcome may be owned by the principal (indicator variable $\left.I^{P}=1\right)$ or by the agent $\left(I^{P}=0\right)$. The former will be the case if the principal is an investor, a landowner, a consumer or a "breather" and the agent correspondingly a manager, a sharecropper, a producer or a 
polluter. Examples of the latter are the insurer-homeowner and the lender-borrower relationships. $I^{P}$ is assumed to be determined by exogenous factors, and is not a variable of choice in the problem.

The probabilities of outcomes and signals are denoted $f(\cdot)$ with arguments indicating which random variables are intended. Hence $f\left(x_{k}\right)$ is the marginal probability of the outcome $x_{k} . f\left(x_{k} \mid y_{i}\right)$ is the conditional probability of $x_{k}$ given the signal $y_{i}$ etc. The expectations operator is denoted $E$. The probability of any outcome-signal combination $(x, y, z)$ is a function of $a-f(x, y, z, a)$. In Section 3 below particular attention will be paid to information systems and outcome functions satisfying the assumption:

Assumption 1. For any values $(x, y, z), f(x, y, z, a)$ is twice differentiable in $a$. The matrix of derivatives of second-order with respect to $a$ is diagonal.

When $a$ is one-dimensional ( $p=1)$, the latter part of Assumption 1 is trivially true. When $p>1$, the assumption implies that the marginal effects on $f$ of the different components of $a$, are independent. When attempting to extend the theory from $p=1$ to $p>1$, this seems to be a natural starting point. ${ }^{5}$

In Section 5 below it is convenient, mainly for expositional reasons, to work from a different assumption about the action space:

\section{Assumption 2. $\mathscr{A}$ is finite.}

Finally, a sharing rule $-S:\{y\} \rightarrow \mathscr{R}-$ is a contract specifying the transfer of $s$ units of money from the principal to the agent, conditional on the signal received from the information system being used. Contracts may only be made conditional on signals which are observed by principal and agent. Preferences are represented by utility functions $U\left(I^{P} x-s\right)$ for the principal and $V\left(\left(1-I^{P}\right) x+s, a\right)$ for the agent to whom decision making is delegated.

Preferences are assumed to satisfy:

Assumption 3. $U$ is trice differentiable, increasing and concave. $V$ is trice differentiable in either argument, increasing and concave in the first argument and concave in the second argument.

Derivatives with respect to the first argument of utility functions are indicated by ('). Derivatives with respect to a are indicated by subscripts. In Sections 3 and 4 a further assumption is made which is subsequently relaxed in Section 5:

Assumption 4. $V(\cdot, \cdot)=V_{1}\left(\left(1-I^{P}\right) x+s\right)+V_{2}(a)$.

The agency information problem may now be defined as follows:

Definition 1. An agency information problem, denoted $\left[X, U, V, \mathscr{H}, I^{P}\right]$, is to choose a pair of functions $(Y, S)$ and an action $a$ to $\max E U\left(I^{P} X-S\right)$ subject to,

$$
\begin{aligned}
& Y \in \mathscr{H} \\
& S:\{y\} \rightarrow \mathscr{R} \\
& a \in \mathscr{A} \\
& E V\left(\left(1-I^{P}\right) X+S, a\right) \geqq \bar{V} \\
& a \in \operatorname{argmax} E V\left(\left(1-I^{P}\right) X+S, a\right) .
\end{aligned}
$$

The agency information problem is a game with a cooperative and a non-cooperative stage. The cooperative stage involves choosing the incentive mechanism $(Y, S)$. It is 
chosen to be Pareto-efficient subject to the agent choosing his own best action in the second stage. Every efficient $(Y, S)$ combination may be generated by properly choosing the agent's level of utility $\bar{V}$.

The agency information problem is a generalization of previous agency models. These may be classified according to assumptions made about the set of information systems $\mathscr{H}$. A discussion is given in the following section.

\section{VALUE OF INFORMATION}

For an agency problem $\left[X, U, V, \cdot, I^{P}\right]$ a ranking of information systems $Y$ may be characterized by

$$
W(Y)=\max _{S} E U\left(I^{P} X-S\right) \quad \text { subject to (2), (3), (4), (5). }
$$

This ranking may be used to define the concept of marginal value of an information system given some information system $Y$ :

Definition 2. $Z$ is said to have marginal value given $Y$ in the agency problem $\left[X, U, V, \cdot, I^{P}\right]$ if $W((Y, Z))>W(Y)$.

The concept of value is that of economic demand value. $Z$ has marginal value if the agency is willing to pay something for the opportunity to base the incentive contract on $Z$ and $Y$ rather than $Y$ alone. If $Z$ has marginal value given $Y^{0}$-no information-, it is simply said to have value in the agency problem.

In this section we shall characterize the set of information systems which have marginal value given an information system $Y$ for a fixed agency problem. A notable feature of this set is that it depends only slightly on the characteristics of the agency problem $(X, U, V)$ when $I^{P}=1$.

Several results in agency theory may be interpreted as value of information propositions. Theorem 11 in Wilson (1968) says that when principal and agent are risk averse, have identically sloped, linear risk-tolerance functions, and the agent has preferences for outcome only ( $V$ is constant in its second argument), then the sharing rule would be a non-constant function of $x$ if $X \in \mathscr{H}$. In other words the information system $X$ is valuable in agency problems with the stated characteristics. In this case the agent and the principal will have identical preferences over actions under optimal risk sharing, and the information system $A$ does not have marginal value given $X$ i.e. there is no need to observe the action as well. ${ }^{6}$

Stiglitz ((1974), Proposition 11) demonstrates that some sharing rule which is linear in $x$, will dominate constant sharing rules when the action (effort) has negative marginal utility to the agent. Hence $X$ is valuable in this case as well. Gjesdal (1976), Harris and Raviv $(1976,1979)$, Holmström $(1977,1979)$ and Shavell (1979) address the problem of marginal value given $X$ in this model. Of particular interest are the results of Holmström and Shavell who characterize the set of information systems which are marginally valuable given $X$ when $\mathscr{A}$ is one-dimensional $(p=1)$. The objective of this section is to generalize these results to an arbitrary given information system $Y$ (and also $p>1$ ). One other work which did not assume that the outcome $X$ is observable, is that of Mirrlees (1976) who discusses the optimal sharing rule when $\mathscr{H}$ consists of information systems which are distributed as $X+\varepsilon$, where $\varepsilon$ is white noise. ${ }^{7}$

An agency problem and some information system $Y$ are then given. The problem is to derive the conditions which an information system $Z$ must satisfy to have marginal value. There are two reasons why this may be the case: $Z$ may improve risk sharing and/or incentives. Two conditions will be formulated each of which is "almost" sufficient to ensure that $Z$ has marginal value. These are the conditions of marginal insurance value and marginal incentive informativeness. 
The following notation will be used. The optimal transfers based on $Y$ alone are denoted $s_{i}\left(\forall i: s_{i}=S\left(y_{i}\right)\right)$. The action induced by this sharing rule is denoted $a^{*}$. The transfers based on $(Y, Z)$ are denoted $s_{i}+s_{i j}\left(\forall i, j: s_{i}+s_{i j}=S\left(y_{i}, z_{j}\right)\right)$. To define marginal insurance value the incentive constraint (5) is ignored.

Definition 3. $Z$ has marginal insurance value given $Y$ in the agency problem $\left[X, U, V, \cdot, I^{P}\right]$ if for some $i$, it is not true that $\forall j, s_{i j}=0$ solves the risk-sharing problem:

subject to

$$
\max _{\left\{s_{i j}\right\}} \sum_{k, j} U\left(I^{P} x_{k}-s_{i}-s_{i j}\right) f\left(x_{k}, y_{i}, z_{j}, a^{*}\right)
$$

$$
\left.\sum_{k, j} V\left(\left(1-I^{P}\right) x_{k}+s_{i}+s_{i j}, a^{*}\right)\right) f\left(x_{k}, y_{i}, z_{j}, a^{*}\right)=\sum_{k} V\left(\left(1-I^{P}\right) x_{k}+s_{i}\right) f\left(x_{k}, y_{i}, a^{*}\right) .
$$

The agency is faced with $m$ risk sharing problems-one for each $i$. $Z$ has marginal insurance value if risk is better shared using a contract based on $Z$ and $Y$, in at least one of these problems. For each risk sharing problem the Lagrangian may be formed, with Lagrange multipliers $\lambda_{i}$. If $s_{i j} \equiv 0$ solves each of these problems, it must satisfy the corresponding first-order conditions. Hence

$$
\forall i, \forall j \quad \sum_{k}\left\{U^{\prime}\left(I^{P} x_{k}-s_{i}\right)-\lambda_{i} V^{\prime}\left(\left(1-I^{P}\right) x_{k}+s_{i}, a^{*}\right)\right\} f\left(x_{k}, y_{i}, z_{j}, a^{*}\right)=0 .
$$

Or, since either $U^{\prime}()$ ) or $V^{\prime}()$ is independent of $x_{k},(7)$ implies that for any $\lambda, \exists$ constants $\bar{G}\left(y_{1}, \lambda, a^{*}\right), \ldots, \bar{G}\left(y_{m}, \lambda, a^{*}\right)$, such that, $\forall i, j$

$$
\begin{aligned}
\sum_{k}\left\{U^{\prime}\left(I^{P} x_{k}-s_{i}\right)-\lambda V^{\prime}\left(\left(1-I^{P}\right) x_{k}+s_{i}, a^{*}\right)\right\} f\left(x_{k} \mid y_{i}, z_{j}, a^{*}\right) & \equiv G\left(y_{i}, z_{j}, \lambda, a^{*}\right) \\
& =\bar{G}\left(y_{i}, \lambda, a^{*}\right) .
\end{aligned}
$$

Optimal risk sharing requires that expected marginal utility should be the same for all signals $z_{j}$. In general the owner's expected marginal utility will be a function of $z$ as well as $y$. When (8) holds, it may, however, be said to be conditionally independent of $z$ given $y$. Note that the relationship between marginal insurance value and marginal value in the agency problem is non-trivial, as insurance value is defined without regard to the incentive effects of the sharing rule.

Next the terms incentive problem and marginal incentive informativeness are defined.

Definition 4. An incentive problem is said to exist given $Y$ for $a_{h}$ at $a^{*}$ if

$$
\left.\frac{\partial E U\left(I^{P} X-S(y)\right)}{\partial a_{n}}\right|_{a=a^{*}} \neq 0 .
$$

The agent's marginal disutility of a change in $a$, given the sharing rule $S(y)$, is 0 by virtue of (5) assuming $a^{*}$ is in the interior of $\mathscr{A}$. If then the principal will derive positive utility from a change in $a$ (in some direction), then $a^{*}$ is not first-best Pareto efficient given the sharing rule $S .^{8}$

When an incentive problem exists given $Y$, a modified contract based on the signals from $Z$ as well, may improve the agent's choice in a Pareto sense provided the following condition is satisfied,

Definition 5. An information system $Z$ is said to be marginally incentive informative given $Y$ for $a_{h}$ at $a^{*}$ if $\nexists$ constants $\bar{H}_{h}\left(y_{1}, a^{*}\right), \ldots, \bar{H}_{h}\left(y_{m}, a^{*}\right)$ such that,

$$
\forall_{j}\left|\frac{\partial f\left(y_{i}, z_{j}, a\right) / \partial a_{h}}{f\left(y_{i}, z_{j}, a^{*}\right)}\right|_{a=a^{*}} \equiv H_{h}\left(y_{i}, z_{j}, a^{*}\right)=\bar{H}_{h}\left(y_{i}, a^{*}\right) .
$$

Holmström (1979) defines marginal incentive informativeness for the case $Y=X$. Following his arguments it is easy to show that if $Z$ is not marginally incentive informative 
given $Y$ for any $a_{h}$ at any value of $a$, then

$$
f(y, z, a)=f(y, z) f(y, a) .
$$

Analogous to the relationship among random variables (11) may be said to express the conditional independence of $a$ and $Z$ given $Y$. Note, however, that $a$ is not a random variable.

The central result on marginal value of information will now be proven, and then interpreted in the light of the concepts just defined.

Proposition 1. Let Assumptions 1, 3 and 4 hold and an agency problem $[X, U, V,\{Y\}, 1]$ be given. Let $s_{i}=S\left(y_{i}\right)$ and $a^{*}$ solve the problem. Assume that $a^{*}$ is unique and in the interior of $\mathscr{A}$. Then $Z$ has marginal value given $Y$ unless

$$
-G\left(y_{i}, z_{j}, a^{*}, \lambda\right)+\sum_{h} \mu_{h} V^{\prime}\left(s_{i}, a^{*}\right) H_{h}\left(y_{i}, z_{j}, a^{*}\right)=0 \quad \text { for a.e. } y_{i}, z_{j},
$$

where

$$
\mu_{h}=\frac{\sum_{k} \sum_{i} U\left(x_{k}-s_{i}\right) f_{a_{h}}\left(x_{k}, y_{i}, a^{*}\right)}{-\left\{\sum_{i} V\left(s_{i}, a^{*}\right) f_{a_{h} a_{h}}+V_{a_{h} a_{h}}\right\}} .
$$

A proof of Proposition 1 is given in the Appendix along with a discussion of the important assumptions imposed on $a^{*}$ and $I^{P}$. An informal discussion of the Proposition follows. Clearly (12) will not hold if there exists a signal $y_{i}$ for which the left-hand side of (12) takes on more than one value i.e. depends non-trivially on $z$. Applying the definitions: $\exists i: G\left(y_{i}, \cdot\right)$ depends on $z$ if $Z$ has marginal insurance value. $\exists i: H_{h}\left(y_{i}, \cdot\right)$ depends on $z$ if $z$ is marginally incentive informative for $a_{h}$, and $\mu_{h} \neq 0$ if an incentive problem exists for $a_{h}$.

To understand (12), it is best viewed as a generalization of the results of Holmström ((1979), Proposition 3-sufficiency) and Shavell ((1979), Proposition 5) for the case $Y=X, p=1$ and $\mu>0$. Clearly, when $Y=X, G$ does not depend on $z$, and $H$ depending on $z$ for some value of $x$, is a sufficient condition for $Z$ to have marginal value. This is the Holmström-Shavell result.

Now assume $Y=X$, but $p>1$. Then

$$
\exists h, i: H_{h}\left(y_{i}, \cdot\right) \text { depends on } z \text {, and } \mu_{h} \neq 0 \text {, }
$$

is not quite sufficient for $Z$ to be marginally valuable. Let this value of $h$ be denoted $h_{1}$. There may exist $h_{2}$ for which $H_{h_{2}}(\cdot)$ depends on $z$ and $\mu_{h_{2}} \neq 0$, such that $\mu_{h_{1}} H_{h_{1}}\left(y_{i}, \cdot\right)+$ $\mu_{h_{2}} H_{h_{2}}\left(y_{i}, \cdot\right)$ does not depend on $z$ for any $y_{i}$.

However, $\mu$ is a characteristic of the agency problem, and $H(\cdot)$ is a characteristic of $Z$. It follows that this alignment will only happen by accident when (13) holds. Hence (13) may be regarded as almost sufficient for $Z$ to have marginal value.

The discussion in the preceding paragraphs generalizes readily to the case where $Y$ is some arbitrary information system except that $G\left(y_{i}, \cdot\right)$ may now depend on $z$ as well. In that case $Z$ may be valuable for improving insurance even when it is useless for incentive purposes. If (11) holds, and $\forall h, i: H_{h}\left(y_{i}, \cdot\right)$ is a constant,

$$
\exists i: G\left(y_{i}, \cdot\right) \text { depends on } z
$$

is sufficient for $Z$ to be marginally valuable. When (13) and (14) hold, it is possible that the incentive and insurance effects cancel out. However, again this must be regarded as an accidental case as (14) depends on the joint distribution of $X, Y, Z$, whereas (13) depends on its derivative with respect to $a$. Hence (14) is almost sufficient for marginal value as well. ${ }^{9}$

Summing up this discussion, it may be concluded that the sufficiency part of the Holmström-Shavell results essentially generalizes to arbitrary given information systems 
$Y$ and multidimensional actions. It has been shown that there exists a marginal incentive informativeness condition which is essentially sufficient for marginal value given $Y$, and which is easily seen to be a generalization of the Holmström-Shavell incentive informativeness condition. However, when $X$ is replaced by $Y$, marginal incentive informativeness is no longer a necessary condition for marginal value as an additional information system $Z$ may be valuable as information about the outcome $x$ as well as the action $a$. To understand this note that the agent's and the principal's ability to share risk depend on the precision with which they can measure the owner's marginal utility ex post. However, the transfer payment is a function only of the (conditional) expected marginal utility (given the signal). This is evident from (7) or (8). (8) then states that the expected marginal utility of the owner is conditionally independent of $Z$ given $Y$. Thus $Z$ provides additional information relevant to risk sharing if and only if (8) is false.

The risk sharing/incentives dichotomy which has been constructed, should not be overstressed as the two sources of demand are closely interrelated. Information systems which satisfy (8) but not (10) may still be used to improve risk sharing. $Y=X$ and $Z=A$ is an obvious example. When $X$ alone is observable, it must be used for incentives as well as insurance. If $a$ is observed as well, a contract based on $a$ takes care of incentives and risk is then shared (efficiently) by means of an outcome based contract. Similarly, information systems which satisfy (10), but not (8) may offer opportunities for incentive improvement.

\section{COMPARISONS OF INFORMATION SYSTEMS}

In Section 3 an information system $Y$ was assumed to have been acquired, and the set of information systems $Z$ which had marginal value given $Y$, was characterized. In this section the problem is to choose either $Z$ or $Y .^{10}$ For a fixed agency problem the ranking $W(\cdot)$ tells which one should be chosen. However, in this section a preference relation is sought which is largely independent of the particular agency problem. If $Z$ is preferred to $Y, Z$ is at least as valuable as $Y$ for every agency problem whose outcome function is measurable with respect to the field generated by a prespecified finite partition $\mathscr{E}=\left\{e_{1}, \ldots, e_{q}\right\}$ of the state space (regardless of $a$ ). In addition the agency problem should satisfy Assumptions $2(\mathscr{A}$ is finite) and 4 (the agent's utility function is linearly separable). Assumption 4 plays an important role although it may be relaxed somewhat. Assumption 2 is invoked mainly to avoid unnecessary mathematical complications. ${ }^{11}$

Three likelihood matrices are defined $(r$ is the cardinality of the set $\mathscr{A})$ :

$$
\begin{aligned}
\Lambda(\mathscr{E}, a) & =\left\{\lambda_{i j}=f\left(y_{j} \mid e_{i}, a\right)\right\} & & q \times m \\
\Lambda(\mathscr{A}) & =\left\{\lambda_{i j}=f\left(y_{j} \mid a_{i}\right)\right\} & & r \times m \\
\Lambda(\mathscr{E}, \mathscr{A}) & =\left\{\lambda_{i j}=f\left(y_{j} \mid(e, a)_{i}\right)\right\} & & r \cdot q \times m
\end{aligned}
$$

The likelihood matrices of another information system $Z$ are similarly defined, and are distinguished by "'”,.

We note that although there is no probability space defined on $\mathscr{A}$, the likelihood matrices $\Lambda(\mathscr{A}), \Lambda(\mathscr{E}, \mathscr{A})$ are well-defined. However, the absence of a measure on $\mathscr{A}$ means that $\Lambda(\mathscr{E}, a)$ depends on $a$. With this exception, $\mathscr{E}$ and $\mathscr{A}$ are symmetrical in the definitions. From Blackwell's theorem (Blackwell (1951)) we know that if we want to compare information systems for decision problems with $\mathscr{E}$-measurable outcome functions, the only problem independent ranking is characterized by the following condition,

Condition $B . \quad \Lambda^{\prime}(\mathscr{E}, a)=\Lambda(\mathscr{E}, a) M(\mathscr{E}, a)$, where $M(\mathscr{E}, a)$ is a $m \times m^{\prime}$ dimensional Markov matrix. ${ }^{12}$ Blackwell's theorem says that $Y$ is preferred to $Z$ for all decision problems if and only if Condition B holds. If Condition B is true, $Z$ is distributed as if it is a garbled version of $Y$, with $M(\cdot)$ as the noise-generating mechanism. Analogy 
suggests generalizing Blackwell's condition as if the state space were $\Theta \times \mathscr{A}$. This conjecture actually works to give,

Proposition 2. Let Assumption 2 and 4 hold. An information system $Y$ is weakly preferred to another information system $Z$ for all agency problems with outcome functions $X(e, a)$ if there exists a $m \times m^{\prime}$ dimensional Markov-matrix $M(\mathscr{E}, \mathscr{A})$ which satisfies

\section{Condition $B A$}

$$
\Lambda^{\prime}(\mathscr{E}, \mathscr{A})=\Lambda(\mathscr{E}, \mathscr{A}) M(\mathscr{E}, \mathscr{A})
$$

The converse is not true.

Proof. Assume $I^{P}=1$. The alternative case is done similarly.

Assume that $Y, Z$ satisfy Condition BA. Let $S^{\prime}(z)$ be an arbitrary sharing rule based on $Z$. The proposition will be proved by showing that there exists a sharing rule $S(y)$ based on $Y$, with the property that the principal prefers $S$ to $S^{\prime}$ whereas the agent is indifferent.

$S$ will be constructed in such a way that the agent is induced to choose the same act under $S$ as he would under $S^{\prime}$.

If the element in the $i$-th row and $j$-th column of $M(\mathscr{E}, \mathscr{A})$ is denoted $m_{i j}, S$ is defined implicitly by,

$$
V_{1}\left(S\left(y_{i}\right)\right)=\sum_{j} V_{1}\left(S^{\prime}\left(z_{j}\right)\right) m_{i j} .
$$

When $S$ is defined this way the agent's objective functions $E V\left(S^{\prime}(z), a\right)$ and $E V(S(y), a)$ are identical functions of $a$, and must therefore induce the same choice:

$$
\begin{aligned}
E V\left(S^{\prime}(z), a\right) & =\sum_{k} \sum_{j}\left\{V_{1}\left(S^{\prime}\left(z_{j}\right)\right)+V_{2}(a)\right\} f\left(z_{j} \mid e_{k}, a\right) f\left(e_{k}, a\right) \\
& =\sum_{k} \sum_{i} \sum_{j}\left\{V_{1}\left(S^{\prime}\left(z_{j}\right)\right)+V_{2}(a)\right\} m_{i j} f\left(y_{i} \mid e_{k}, a\right) f\left(e_{k}, a\right) \\
& =\sum_{k} \sum_{i}\left\{V_{1}\left(S\left(y_{i}\right)\right)+V_{2}(a)\right\} f\left(y_{i} \mid e_{k}, a\right) f\left(e_{k}, a\right) \\
& =E V(S(y), a) .
\end{aligned}
$$

The second equality is Condition BA. The third follows by the definition of $S$.

The identity of objective functions also implies that the agent is indifferent between $S$ and $S^{\prime}$. It remains to be proved that the principal prefers $S$ to $S^{\prime}$. Since both sharing rules induce the same choice, the action variable may be suppressed,

$$
\begin{aligned}
E U\left(X-S^{\prime}(z)\right) & =\sum_{k} \sum_{i} \sum_{j} U\left(x_{k}-S^{\prime}\left(z_{j}\right)\right) m_{i j} f\left(y_{i}, e_{k}\right) \\
& \leqq \sum_{k} \sum_{i} U\left(x_{k}-\sum_{j} S^{\prime}\left(z_{j}\right) m_{i j}\right) f\left(y_{i}, e_{k}\right) \\
& \leqq \sum_{k} \sum_{i} U\left(x_{k}-S\left(y_{i}\right)\right) f\left(y_{i}, e_{k}\right) \\
& =E U(X-S(y))
\end{aligned}
$$

where the first inequality follows by Jensen's inequality. The second is derived from the definition of $S$ :

The concavity of $V_{1}$ implies that

$$
S\left(y_{i}\right)<\sum_{j} S^{\prime}\left(z_{j}\right) m_{i j} \text { for every } i .
$$

To prove that the converse is not true, a counterexample is constructed for the case where there are two events $\left\{e_{1}, e_{2}\right\}$ and two actions $\left\{a_{1}, a_{2}\right\}$ : Information systems are defined by means of partitions on the product space $\Theta \times \mathscr{A}$ :

$$
\begin{aligned}
& Y:=\left\{\left\{\left(e_{1}, a_{1}\right)\left(e_{2}, a_{2}\right)\right\},\left\{\left(e_{1}, a_{2}\right)\right\},\left\{\left(e_{2}, a_{1}\right)\right\}\right\} \\
& Z:=\left\{\left\{\left(e_{1}, a_{1}\right)\right\},\left\{\left(e_{1}, a_{2}\right)\right\},\left\{\left(e_{2}, a_{1}\right)\right\},\left\{\left(e_{2}, a_{2}\right)\right\}\right\} .
\end{aligned}
$$


$Z$ is perfect information. $Y$ is strictly coarser than perfect information, and hence Condition BA does not hold. However, $Y$ is always as valuable as $Z$. To see this assume neither $e_{1}$ nor $e_{2}$ are null-sets (if they are, the claim is obvious). Then $a_{1}$ may be enforced by a contract based on $Y$ which penalizes the agent sufficiently if $\left\{\left(e_{1}, a_{2}\right)\right\}$ obtains. Similarly $a_{2}$ may be enforced by a penalty if $\left\{\left(e_{2}, a_{1}\right)\right\}$ obtains. This enforcement is costless since if the agent complies, no penalty will be imposed. Given this enforcement, $Y$ is equivalent to perfect information since it is perfect information on events for a given $a$. It follows that $Y$ is as valuable as $Z$ for any agency information problem with $\mathscr{E}$-measurable outcome function.

In the agency problem information is used for insurance and incentive purposes. It is instructive to see how the information system $Y$ solves each problem at least as well as $Z$. If $m_{i j}$ is interpreted as the conditional probability $f\left(z_{j} \mid y_{i}\right)$, then $S\left(y_{i}\right)$ may be interpreted as the certainty equivalent of the gamble which pays $S^{\prime}\left(z_{j}\right)$ with probability $f\left(z_{j} \mid y_{i}\right)$. Hence $S$ may be viewed as employing a deductible $E\left(S^{\prime} \mid y\right)-S(y)$ for every $y$, rather than randomizing through this gamble. ${ }^{13}$ The same incentive effect is obtained, and less risk is imposed on the agent.

The fact that $Z$ in the counterexample of Proposition 2 is perfect information, is not essential to the argument. In fact $\left\{a_{1}, a_{2}\right\}$ may alternatively be interpreted as some two-set partition of the action space $\mathscr{A} .^{14}$ Adopting this interpretation, the two subsets may be denoted $\bar{a}_{1}$ and $\bar{a}_{2}$. With $Z$ the agency is then limited to enforcing the agent's most preferred choice in either $\bar{a}_{1}$ or $\bar{a}_{2}$. With $Y$ the agency can do as well. The idea is that for incentive purposes an information system which may detect some shirking (whether the agent chooses an action in $\bar{a}_{1}$ rather than $\bar{a}_{2}$ or vice versa) may be replaced by any information system which detects this shirking with positive probability. ${ }^{15}$ This argument shows how the game nature of the agency problem influences the comparison of information systems. It is not valid to treat the action $a$ as if it were a state variable in some Bayesian decision problem.

In this first part of the sufficiency proof the full strength of Condition BA is not needed. Use is only made of the weaker Condition A which may be identified with yet another Markov matrix, $M(\mathscr{A})$.

\section{Condition A. $\Lambda^{\prime}(\mathscr{A})=\Lambda(\mathscr{A}) M(\mathscr{A})$.}

In fact one might as well have summed over $k$ to eliminate the event variable altogether in this part of the proof. Similarly, if Condition A is true, the agent may be induced to make the same choice with $Y$ as with $Z$ without being worse off, in the case where he owns $X$ but is risk neutral. In the latter case as well as in the case where the principal owns $X$ and is risk neutral, the functional inequality $E\left(S^{\prime} \mid y\right) \geqq S(y)$ is sufficient to make the principal prefer $S$ to $S^{\prime}$ given that both induce the same act. Finally, Condition $A$ is also sufficient when we only consider outcome functions which are measurable with respect to $(\varnothing, \Theta)$ for every $a$, i.e. non-stochastic outcome functions. In that case Conditions $\mathrm{A}$ and $\mathrm{BA}$ are equivalent. Thus;

\section{Corollary 1. Assume Condition A holds, and either}

(i) the owner is risk neutral, or

(ii) $X(\theta, a)$ is measurable with respect to $(\varnothing, \Theta)$

then $Y$ is weakly preferred to $Z$.

Since (i) and (ii) are cases where there is no risk-sharing demand for information, the corollary justifies defining Condition $A$ as the incentive ranking of information systems. ${ }^{16}$ This result seems to correspond to the idea of responsibility accounting: "For example, the (performance) report for a shop foreman's department would contain only his controllable costs. (Horngren [1977], p. 161)." 
In the second part of the proof of sufficiency, Condition B is used. To prove that the principal prefers $S$, it must be demonstrated that $S^{\prime}$ does not insure the principal's risk represented by $X$, better than $S$. Again arguing as if $M$ is the conditional distribution of $Z$ given $Y$, it is clear that $S^{\prime}$ cannot be better for risk sharing since the randomization represented by $Z$ is independent of $e$, and hence of $X$.

It follows then, as another corollary of the proposition, that insurance schemes should not be randomized-hardly a surprising result. The idea of randomized incentive schemes may seem as far-fetched to some. However, it is a fact that the set of admissible utility functions must be restricted in the Proposition. Additive utility functions make $S$ independent of $a$ in (15) ${ }^{17}$ Under more general assumptions randomization may well be Pareto-optimal. This problem is explored in the following section.

The need to impose restrictions on utility functions is another proof that the agency information problem is not, formally, a Bayesian decision problem. It follows that Proposition 2 is not a trivial generalization of Blackwell's theorem. The agency problem contains restrictions which are not of the form encountered in decision problems. The possibility of randomized schemes, moreover, proves that there is no way to reinterpret or reformulate the problem, to make it formally equivalent to a decision problem.

\section{RANDOM INCENTIVE SCHEMES}

Proposition 2 does not hold for arbitrary utility functions $V(\cdot)$. In this section it will be demonstrated that there are utility functions for which $Y$ may be strictly more valuable than $Z$ even though $Z$ is ranked higher by the Condition BA ordering. In other words randomization may be efficient. ${ }^{18}$ For such a demonstration, the simplest case will suffice. $Z$ is therefore initially assumed to be the null system $Y^{0}$, and $Y^{\prime}$ 'some random mechanism. ${ }^{19}$ First an example is offered and discussed. The section is concluded with a look at the general case.

Example. The agent's utility function is,

$$
V(s, a)=s(4-a)-\frac{s^{2}}{a},
$$

defined on the set

$$
\left\{0<a<4,0 \leqq s \leqq \frac{a}{2}(4-a)\right\} .
$$

Note that the agent is risk averse on this domain.

For a given $s$, the agent's optimal action is found by solving,

$$
-s+\frac{s^{2}}{a^{2}}=0 \Rightarrow a=\sqrt{s}\left(s<\frac{16}{9}\right)
$$

as the second order condition for a maximum is satisfied.

The principal is risk neutral and the outcome function is $x=a$. No information is available. Hence the principal maximizes,

$$
U=a-s=\sqrt{s}-s
$$

and the solution is $s=\frac{1}{4}, a=\frac{1}{2}, U=\frac{1}{4}, V=\frac{3}{4} . \frac{3}{4}$ is assumed to be an acceptable level of utility for the agent.

We shall now demonstrate that a random sharing rule will make both individuals better off:

$$
S^{\prime}=\left\{\begin{array}{l}
s_{1} \text { with probability } \frac{1}{2} \\
s_{2} \text { with probability } \frac{1}{2}
\end{array}\right.
$$


With this sharing rule the agent's first-order condition becomes

$$
2 E V_{a}^{\prime}=-s_{1}+\frac{s_{1}^{2}}{a^{2}}-s_{2}+\frac{s_{2}^{2}}{a^{2}}=0
$$

which gives

$$
a^{2}=\frac{s_{1}^{2}+s_{2}^{2}}{s_{1}+s_{2}} .
$$

If $s_{1}$ is arbitrarily fixed at $0, a=\sqrt{s_{2}}$ and

$$
\begin{aligned}
& E U=\sqrt{s_{2}}-\frac{1}{2} s_{2}=\frac{1}{2} \\
& E V=\frac{1}{2}\left[s_{2}\left(4-\sqrt{s_{2}}\right)-\frac{s_{2}^{2}}{\sqrt{s_{2}}}\right]=1
\end{aligned}
$$

at the principal's optimum $s_{2}=1$. Hence both are better off with randomization.

The key to understanding the example is to observe that the agent's first-order condition (16) is convex in $s$. This implies that making the salary random will increase the agent's output. To interpret convexity the second-derivative of $(16)$ with respect to $s\left(V_{a}^{\prime \prime}\right)$ may be expressed by means of the agent's absolute risk aversion $\left(R_{A}\right)$ :

$$
V_{a}^{\prime \prime}=-V^{\prime} \frac{d R_{A}}{d a}-R_{A} V_{a}^{\prime} .
$$

The benefit to the principal of giving away an extra non-random $\$$ has the same sign as $V_{a}^{\prime}$. Hence $V_{a}^{\prime}>0$ at the optimal non-random $s$. It follows that (16) is convex $\left(V_{a}^{\prime \prime}>0\right)$ only if $d R_{A} / d a<0$. Hence randomizing the agent's salary makes him work harder because hard work increases his risk tolerance. The second incentive effect$R_{A} V_{a}^{\prime}$-may be called the "utility effect". Risk lowers the agent's level of utility which influences his action preferences. The "utility effect" on $a$ is negative as $V_{a}^{\prime}>0$, and it is (not surprisingly) proportional to the agent's absolute risk aversion.

What is more surprising, is that in the setting of the example-risk neutral principal, non-binding efficiency constraint (4) and no information-a positive incentive effect $\left(V_{a}^{\prime \prime}>0\right)$ is also a sufficient condition for randomization to be efficient. ${ }^{20}$ The explanation is as follows. Consider increasing the agent's salary by a small amount. The agent is certainly better off. At the optimum the principal is indifferent as the cost equals the benefit. Now make the extra salary random, but such that its mean is equal to the non-random raise being considered. ${ }^{21}$ The randomization does increase the risk imposed on the agency. However, at the margin the agent is strictly better off as long as the expected salary increase is positive. The principal is risk neutral, and the cost of the random increase is therefore identical to that of a non-random one. However, the benefit of a random raise is larger, since its incentive effect is greater. Hence the principal is strictly better off as well.

The discussion in the preceding paragraph does not generalize. That (4) is nonbinding and the principal is risk neutral, is crucial to the argument. In the general case the efficiency of randomization hinges on a trade-off between an incentive effect which may be positive, and a risk effect which is always non-positive, and is a function of the agency's risk-aversion. The latter concept, which is derived from the risk-aversion of the participants, is defined below and denoted $R$. The main result on randomization as an incentive mechanism may then be summed up as follows.

Proposition 3. Assume $I^{P}=p=1, \mu>0$, and that the agent's choice with the optimal deterministic sharing rule- $a^{*}-i$ unique and in the interior of $\mathscr{A}$. Then the transfer 
payment should be randomized when signal $y_{i}$ obtains, if

$$
\frac{1}{R\left(y_{i}\right)} \frac{\partial R_{A}\left(y_{i}\right)}{\partial a}<-\frac{1}{\mu} \frac{E\left\{U^{\prime}(\cdot) \mid y_{i}\right\}}{V^{\prime}\left(S\left(y_{i}\right)\right)} .
$$

Proof. The information system $Y$ is given. The optimal non-random transfer payment is $S^{*}\left(y_{i}\right)=s_{i}^{*}$ when the signal is $y_{i}$, and this sharing rule induces the agent to choose $a^{*}$. It is also assumed that the principal owns $X$, and at the optimum wants the agent to choose a larger $a$ (which is a scalar).

Assumptions 1 and 3 imply that the agent's objective function is differentiable. Hence any interior maximum must satisfy

$$
\frac{\partial E V(S, a)}{\partial a}=0
$$

For $j \neq i$, let $s_{j}$ be fixed at $s_{j}^{*}$, and assume that $a^{*}$ is a unique interior maximum. Then (20) defines a differentiable function $a\left(s_{i}\right)$ at least in a neighbourhood of $s_{i}^{*}$. Since $s_{i}^{*}$ maximizes

$$
L=E U(X-S)+\lambda E V\left(S, a\left(s_{i}\right)\right),
$$

it satisfies the first- and second-order conditions (using (20)):

$$
\begin{gathered}
\frac{d L}{d s_{i}}=\frac{\partial E U}{\partial s_{i}}+\lambda \frac{\partial E V}{\partial s_{i}}+\frac{\partial E U}{\partial a} \frac{d a}{d s_{i}}=0 \\
\frac{d^{2} L}{d s_{i}^{2}}=\frac{\partial^{2} E U}{\partial s_{i}^{2}}+\lambda \frac{\partial^{2} E V}{\partial s_{i}^{2}}+\frac{\partial^{2} E U}{\partial s_{i} \partial a} \frac{d a}{d s_{i}}+\lambda \frac{\partial^{2} E V}{\partial s_{i} \partial a} \frac{d a}{d s_{i}}+\frac{\partial^{2} E U}{\partial a^{2}}\left(\frac{d a}{d s_{i}}\right)^{2} \\
+\frac{\partial^{2} E U}{\partial a \partial s_{i}} \frac{d a}{d s_{i}}+\frac{\partial E U}{\partial a} \frac{d^{2} a}{d s_{i}^{2}}+\lambda \frac{\partial^{2} E V}{\partial a^{2}}\left(\frac{d a}{d s_{i}}\right)^{2}+\lambda \frac{\partial^{2} E V}{\partial a \partial s_{i}} \frac{d a}{d s_{i}}<0 .
\end{gathered}
$$

The derivatives of $a\left(s_{i}\right)$ are found by differentiating implicitly with respect to $s_{i}$ in (21):

$$
\begin{gathered}
\frac{d a}{d s_{i}}=\frac{\partial^{2} E V / \partial a \partial s_{i}}{-\partial^{2} E V / \partial a^{2}}=\frac{V_{a}^{\prime} f+V^{\prime} f_{a}}{-\partial^{2} E V / \partial a^{2}} \\
\frac{d^{2} a}{d s_{i}^{2}}=\frac{d\left[\partial^{2} E V / \partial a \partial s_{i}\right] / d s_{i}+d\left[\partial^{2} E V / \partial a^{2}\right] / d s_{i} d a / d s_{i}}{-\partial^{2} E V / \partial a^{2}} .
\end{gathered}
$$

Using,

$$
\mu=\partial E U / \partial a /-\partial^{2} E V / \partial a^{2}>0
$$

and substituting (23) into (21), the following expression is derived for later use,

$$
f\left(y_{i}\right)\left(\lambda+\mu \frac{f_{a}\left(y_{i}\right)}{f\left(y_{i}\right)}\right)=-\frac{1}{V^{\prime}\left(s_{i}^{*}, a^{*}\right)}\left(\frac{\partial E U}{\partial s_{i}}+\mu V_{a}^{\prime}\left(s_{i}^{*}, a^{*}\right) f\left(y_{i}\right)\right) .
$$

Now consider spinning a roulette wheel or using some other random mechanism to determine the agent's pay when $Y=y_{i}$. The random payment, which is independent of $X$ and $a$, is denoted $T$. Using $I\left(y_{i}\right)$ for the indicator random variable of the $\operatorname{set}\left\{\theta: Y=y_{i}\right\}$, the following incentive scheme is proposed:

$$
S^{*}+h T I\left(y_{i}\right)
$$

where $h$ is a scalar. The problem is to maximise

$$
L(h, T)=E U\left(X-S^{*}-h T I\left(y_{i}\right)\right)+\lambda E V\left(S^{*}+h T I\left(y_{i}\right), a\right)
$$

with respect to $h$ and $T$ subject to the incentive constraint.

First $T$ is considered fixed, and the conditions under which this randomization is (locally) useful will be derived. Those are the conditions under which $h=0$ is not a local maximum of $L(h, T)$. Then the choice of $T$ is considered. 
Differentiating $L(\cdot)$ with respect to $h$ at $h=0$ is considerably simplified by noting that $\partial E U / \partial h=\partial E U / \partial s_{i} E T$

and

$$
\partial^{2} E U / \partial h^{2}=\partial^{2} E U / \partial s_{i}^{2} E T^{2} \quad \text { etc. }
$$

at $h=0$.

$$
\begin{gathered}
\frac{d L}{d h}=\frac{\partial E U}{\partial s_{i}} E T+\lambda \frac{\partial E V}{\partial s_{i}} E T+\frac{\partial E U}{\partial a} \frac{d a}{d h} \\
\frac{d^{2} L}{d h^{2}}=\frac{\partial^{2} E U}{\partial s_{i}^{2}} E T^{2}+\lambda \frac{\partial^{2} E V}{\partial s_{i}^{2}} E T^{2}+\left(\frac{\partial^{2} E U}{\partial s_{i} \partial a}+\lambda \frac{\partial^{2} E V}{\partial s_{i} \partial a}\right) \frac{d a}{d h} E T \\
+\frac{\partial^{2} E U}{\partial a^{2}}\left(\frac{d a}{d h}\right)^{2}+\frac{\partial^{2} E U}{\partial a \partial s_{i}} \frac{d a}{d h} E T+\frac{\partial E U}{\partial a} \frac{d^{2} a}{d h^{2}} \\
+\lambda \frac{\partial^{2} E V}{\partial a^{2}}\left(\frac{d a}{d h}\right)^{2}+\lambda \frac{\partial^{2} E V}{\partial a \partial s_{i}} \frac{d a}{d h} E T .
\end{gathered}
$$

Differentiating twice in the agent's first order condition,

$$
\begin{gathered}
\frac{d a}{d h}=\frac{\frac{\partial^{2} E V}{\partial a \partial s_{i}}}{-\frac{\partial^{2} E V}{\partial a^{2}}} E T=\frac{d a}{d s_{i}} E T \\
\frac{d^{2} a}{d h^{2}}=\frac{1}{-\partial^{2} E V / \partial a^{2}}\left[\frac{\partial^{3} E V}{\partial a \partial s_{i}^{2}} E T^{2}+2 \frac{\partial^{3} E V}{\partial a^{2} \partial s_{i}}(E T)^{2} \frac{d a}{d s_{i}}+\frac{\partial^{3} E V}{\partial a^{3}}\left(\frac{d a}{d s_{i}}\right)^{2}(E T)^{2}\right] \\
=(E T)^{2} \frac{d^{2} a}{d s_{i}^{2}}+\frac{\partial^{3} E V / \partial a \partial s_{i}^{2}}{-\partial^{2} E V / \partial a^{2}}\left(E T^{2}-(E T)^{2}\right) .
\end{gathered}
$$

Substituting (29) and (30) into (27) and (28),

$$
\begin{gathered}
\frac{d L}{d h}=E T \frac{d L}{d s_{i}}=0 \\
\frac{d^{2} L}{d h^{2}}=\frac{d^{2} L}{d s_{i}^{2}}(E T)^{2}+\left[\frac{\partial^{2} E U}{\partial s_{i}^{2}}+\lambda \frac{\partial^{2} E V}{\partial s_{i}^{2}}+\mu \frac{\partial^{3} E V}{\partial a \partial s_{i}^{2}}\right]\left(E T^{2}-(E T)^{2}\right)
\end{gathered}
$$

since

$$
\frac{\partial^{3} E V}{\partial a \partial s_{i}^{2}}=V^{\prime \prime}\left(s_{i}^{*}, a^{*}\right) f_{a}+V_{a}^{\prime \prime}\left(s^{*}, a^{*}\right) f
$$

using (26) yields,

$$
\begin{aligned}
\frac{d^{2} L}{d h^{2}} & =\frac{d^{2} L}{d s_{i}^{2}}(E T)^{2}+\left[\frac{\partial^{2} E U}{\partial s_{i}^{2}}+V^{\prime \prime}\left(s_{i}^{*}, a^{*}\right) f\left(\lambda+\mu \frac{f_{a}}{f}\right)+\mu V_{a}^{\prime \prime}\left(s_{i}^{*}, a^{*}\right) f\right] \cdot\left(E T^{2}-(E T)^{2}\right) \\
& =\frac{d^{2} L}{d s_{i}^{2}}(E T)^{2}+\left[\frac{\partial^{2} E U}{\partial s_{i}^{2}}-\frac{V^{\prime \prime}}{V^{\prime}}\left(\frac{\partial E U}{\partial s_{i}}+\mu V_{a}^{\prime} f\right)+\mu V_{a}^{\prime \prime} f\right]\left(E T^{2}-(E T)^{2}\right) \\
& =\frac{d^{2} L}{d s_{i}^{2}}(E T)^{2}+\left[\frac{\partial^{2} E U}{\partial s_{i}^{2}}-\frac{V^{\prime \prime}}{V^{\prime}} \frac{\partial E U}{\partial s_{i}}+\mu f\left(-\frac{V^{\prime \prime}}{V^{\prime}} V_{a}^{\prime}+V_{a}^{\prime \prime}\right)\right]\left(E T^{2}-(E T)^{2}\right) \\
& =\frac{d^{2} L}{d s_{i}^{2}}(E T)^{2}+\left[\frac{\partial E U}{\partial s_{i}} \cdot\left(\frac{\partial^{2} E U}{\partial s_{i}^{2}} / \frac{\partial E U}{\partial s_{i}}-\frac{V^{\prime \prime}}{V^{\prime}}\right)-\mu f V^{\prime} \frac{d R_{A}}{d a}\right] \operatorname{var} T .
\end{aligned}
$$


Since $d L / d h=0, L$ has a local maximum at $h=0$ unless $d^{2} L / d h^{2}>0$. The first term in (33) is non-positive because of (22). It follows that $\partial^{2} L / \partial h^{2}>0$ only if the term inside the square bracket, where the term inside the parenthesis is defined to be the agency's (absolute) risk aversion $(R)$, is positive. This is (19).

On the other hand if the term in the square bracket is positive, and taking $T$ to be a choice variable, $d^{2} L / d h^{2}$ can be made positive by choosing a $T$ which has sufficiently large variance in relation to its mean. This proves Proposition 3.

Several remarks on the analysis are in order. First, note that the results are consistent with the discussion of the example. There $U^{\prime \prime}=\lambda=0$, and from (32) it follows that randomization is efficient whenever the agent's first-order condition is convex.

Secondly, attention is called to a difference between this analysis and the analysis in Section 3. There it was demonstrated that an information system is marginally valuable whenever it provides information about a not already available. The increased risk imposed on the agency by the revised contract does not matter (at the margin). The reason for this is that the incentive effect of more information is a first-order effect (influences the first derivative of the objective function) whereas the risk effect is of second order (influences only the second derivative). However, the incentive effect of randomization is a second order effect as well. It follows that randomization is valuable only if the incentive effect is "large enough" relative to the negative effect of more risk.

Thirdly, it should be clear from (33) that (19) is necessary as well as sufficient for a marginal randomization to effect a Pareto improvement.

Finally, Proposition 3, like Proposition 1, depends on the assumption that $a^{*}$ is unique. If the agent is indifferent between several actions, a marginal randomization may make him jump to an action where risk is less harmful. This may make the principal worse off as long as the agent, when indifferent, chooses the principal's preferred action. To rule out jumps when $a^{*}$ is not unique, it is sufficient to assume that $\partial E V / \partial a$ is uniformly convex i.e. that $\partial^{3} E V / \partial a \partial s_{i}^{2}>0$ for all $a$.

\section{CONCLUDING REMARKS}

In this paper we have tried to develop a theory of incentive-insurance demand for information.

We have shown that information may be used to control decentralized decisionmaking if the probabilities of the signals received from the information system are influenced by the choices made by decision makers. Even though they in general increase social risk, incentive schemes based on information systems which are informative in this sense, will give Pareto-improvement when the choices, that would have been made with no such scheme, are inefficient (except for some special cases). Our results are easily seen to be generalizations of those of Holmström (1979) and Shavell (1979).

As in Holmström and Shavell, it has been assumed that the agent's optimal action is unique. The justification for this assumption as well as the consequences of relaxing it, are important topics for further research.

We have also characterized an informativeness ranking which may be viewed as a generalization of Blackwell's ranking of information systems. It is not, however, a trivial extension of Blackwell's theorem. The incentive problem is a non-cooperative game, and the Bayesian theory of decision making use of information does not apply. Indeed it is shown that more information according to the Blackwell ranking is sufficient but not necessary for an information system to be uniformly as valuable as another system.

There are even cases in which randomization is efficient. In these cases less information (again in the Blackwell sense) is preferred to more, and randomization may be viewed as throwing away information. Risk has a positive incentive effect if the agent's risk aversion decreases when $a$ changes in a direction which is preferred by the principal. 


\section{APPENDIX}

Proof of Proposition 1. $Z$ has no marginal value if and only if the optimal sharing rule based on $Z$ and $Y$ does not depend on $z$ i.e. $a^{*}$ and $\forall i, j: s_{i j}=0$ solves the agency problem. It will be demonstrated that unless $(12)$ is true, $s_{i j} \equiv 0$ does not satisfy a first-order condition of the agency problem. The problem of choosing the sharing rule $S$ for the information system $(Y, Z)$ may be formulated as follows: Max with respect to $s_{i j}$, $a$

$$
\sum_{k} \sum_{i} \sum_{j}\left\{U\left(x_{k}-s_{i}-s_{i j}\right)+\lambda V\left(s_{i}+s_{i j}, a\right)\right\} f\left(x_{k}, y_{i}, z_{j}, a\right)
$$

subject to (2), (3) and (5).

The strategy of the proof is to derive the function $a\left(s_{11}, \ldots, s_{m m^{\prime}}\right)$ in the neighbourhood of $a^{*}$, and use this function to show that (A.1) has no stationary point at $a^{*}, s_{i j} \equiv 0$ unless (12) is satisfied.

Because of differentiability Assumptions 1 and 3, and since $a^{*}$ solves (5) when $s_{i j} \equiv 0$, and is in the interior of $\mathscr{A}: \forall h$;

$$
\left.\frac{\partial}{\partial a_{h}} \sum_{k} \sum_{i} \sum_{j} V\left(s_{i}+s_{i j}, a\right) f\left(x_{k}, y_{i}, z_{j}, a\right)\right|_{a=a^{*}, s_{i j}=0}=0 .
$$

Moreover, since $a^{*}$ is a unique global maximum, the implicit functions theorem implies the existence of a differentiable function $a\left(s_{11}, \ldots, s_{m m^{\prime}}\right)$ in some neighbourhood of $s_{i j} \equiv 0$ (assuming the matrix of second-order conditions corresponding to (A.2) is nonsingular). The derivatives are found by differentiating implicitly with respect to $s_{i j}$ in (ii). Evaluated at $a^{*}, s_{i j} \equiv 0$, the derivatives are,

$$
\begin{gathered}
V^{\prime}\left(s_{i}, a\right) f_{a_{h}}\left(y_{i}, z_{j}, a\right)+\sum_{k}\left\{\sum_{i} \sum_{j}\left(V\left(s_{i}, a\right) f_{a_{h} a_{k}}\left(y_{i}, z_{j}, a\right)+V_{a_{h} a_{k}}\right)\right\} \frac{\partial a_{k}}{\partial s_{i j}}=0 \\
h=1, \ldots, p, \quad i=1, \ldots, m, \quad j=1, \ldots, m^{\prime} .
\end{gathered}
$$

Additivity assumptions on $f(\cdot)$ and $V(\cdot)$ (Assumptions 1 and 4) allow the following simple solution to the simultaneous equation system (A.3); $\forall i, j, h$

$$
\left.\frac{\partial a_{h}}{\partial s_{i j}}\right|_{a=a^{*}, s_{i j}=0}=-\frac{V^{\prime}\left(s_{i}, a\right) f_{a_{h}}\left(y_{i}, z_{j}, a\right)}{\sum_{i} V\left(s_{i}, a\right) f_{a_{h} a_{h}}\left(y_{i}, a\right)+V_{a_{h} a_{h}}} .
$$

Finally, differentiating (A.1) using (A.2), then (A.4) and assuming $\left(y_{i}, z_{i}\right)$ is not a null-set, yields (with again all derivatives evaluated at $a=a^{*}, s_{i j} \equiv 0$, and derivatives involving $a$ interpreted as vectors): $\forall i, j$;

$$
\begin{aligned}
\frac{\partial E U}{\partial s_{i j}}+ & \frac{\partial E U}{\partial a} \frac{\partial a}{\partial s_{i j}}+\lambda \frac{\partial E V}{\partial s_{i j}}+\lambda \frac{\partial E V}{\partial a} \frac{\partial a}{\partial s_{i j}} \\
= & \frac{\partial E U}{\partial s_{i j}}+\lambda \frac{\partial E V}{\partial s_{i j}}+\frac{\partial E U}{\partial a} \frac{\partial a}{\partial s_{i j}} \\
= & f\left(y_{i}, z_{j}, a\right)\left\{-\left[\sum_{k}\left\{U^{\prime}\left(x_{k}-s_{i}\right)-\lambda V^{\prime}\left(s_{i}, a\right)\right\} f\left(x_{k} \mid y_{i}, z_{j}, a\right)\right]\right. \\
& \left.+\sum_{h}\left[\frac{\sum_{k} \sum_{i} U\left(x_{k}-s_{i}\right) f_{a_{h}}\left(x_{k}, y_{i}, a\right)}{-\left\{\sum_{i} V\left(s_{i}, a\right) f_{a_{h} a_{h}}+V_{a_{h} a_{h}}\right\}}\right]\left[V^{\prime}\left(s_{i}, a\right) \frac{f_{a_{h}}\left(y_{i}, z_{j}, a\right)}{f\left(y_{i}, z_{j}, a\right)}\right]\right\} .
\end{aligned}
$$

Unless $(\mathrm{A} .5) \equiv 0, s_{i j} \equiv 0$ is not a stationary point, and $Z$ has marginal value given $Y$. \|

The Appendix will be concluded with a discussion of three assumptions made in Proposition 1: that $a^{*}$ is unique and interior in $\mathscr{A}$, and that the principal owns $X\left(I^{P}=1\right)$.

First, the role played by the assumption that the principal owns $X\left(I^{P}=1\right)$ in Proposition 1, should be pointed out. Mainly, this assumption has been made for expositional reasons. The alternative case $\left(I^{P}=0\right)$ could be developed along the same 
lines. However, one of the striking features of Proposition 1, the fact that marginal incentive informativeness is essentially independent of the agency problem $(X, U, V)$, is lost. When $I^{P}=0,(10)$ is replaced by:

$$
\left.\sum_{k} V^{\prime}\left(x_{k}+s_{i}, a\right) f_{a_{h}}\left(x_{k}, y_{i}, z_{j}, a\right)\right|_{a=a^{*}}=\overline{\bar{H}}\left(y_{i}, a^{*}\right) f\left(y_{i}, z_{j}, a^{*}\right) .
$$

The assumptions imposed on $a^{*}$, on the other hand, are crucial at least technically. Non-interior solutions may surface in some formulations of the agency problem, notably those which assume that $V(\cdot)$ is monotone decreasing in $a$ (effort). If it is assumed that $V(\cdot)$ increases in $a$ for sufficiently low $a$ 's (arguably a more attractive assumption), non-interior solutions are less likely to be a problem. The proof of Proposition 1 relies heavily on the fact that when $a^{*}$ is an interior solution, there exist small variations in the sharing rule which have positive incentive effects while the marginal increase in risk has zero value. When $a^{*}$ is not an interior point, quite large changes in the sharing rule may be required to induce changes in $a$, and even if the incentive effect is positive, it may be outweighted by the non-marginal increase in risk.

Finally, as Mirrlees (1975) has shown, the methods which have been developed to solve for the optimal sharing rule in the agency problem, may not capture the optimal solution unless its induced action is unique (Grossman and Hart (1981) has solved this problem for finite $\mathscr{A}$ ). As it is impossible to check this requirement in advance, Mirrlees' point is quite serious. When the objective is to characterize the set of marginally valuable information systems, non-uniqueness presents a somewhat different problem. As the information system $Y$ and the sharing rule $S(y)$ are given, it is at least possible to check whether these induce a unique action. However, the characterization problem for the case of non-unique $a^{*}$ remains. The difficulty is the following. Assume that the agent given $S(y)$ is indifferent between $a^{\prime}$ and $a^{\prime \prime}$, but chooses $a^{\prime \prime}$ which, although it is not a stationary point of the principal's evaluation measure, is preferred by him to $a^{\prime}$. Unless (10) is satisfied at $a^{\prime \prime}$, it is possible to proceed as above and construct a variation in the sharing rule based on $Z$, which makes some $a^{\prime \prime \prime}$, preferred by the principal to $a^{\prime \prime}$, a local maximum of the agent's evaluation measure instead of $a^{\prime \prime}$. However, it may be that the global solution to the agent's problem is somewhere in the neighbourhood of $a^{\prime}$ for every such variation, thus making the principal worse off. It is necessary to check whether a local improvement is a global improvement as well.

\section{First version received September 1980; final version accepted January 1982 (Eds.).}

This paper is an abridged version of a paper which was presented at the Fourth World Congress of the Econometric Society in Aix-en-Provence Aug. 28th-Sept. 2nd 1980. It is, in part, based on Chapter 4 of my Ph.D. dissertation, "Stewardship Accounting: Controlling Informational Externalities", Graduate School of Business, Stanford University. I gratefully acknowledge the contributions of my principal research advisor Professor Joel S. Demski. This work has also benefited from the criticism and suggestions of Professors John Christensen, Steinar Ekern, Charles Holloway, Thore Johnsen, David Ng, Robert Wilson as well as Oliver Hart and two anonymous referees. My research has been supported by a generous grant from the Norwegian Research Council for Science and the Humanities.

\section{NOTES}

1. Why the principal is affected by the agent's decision, is outside the scope of this paper. In general terms the reason may be that the principal insures the agent's property, that the principal as owner of property is delegating decision-making to the agent because of the agent's comparative advantage, or it may be that the principal (government) is entitled to some share of the agent's property and income. (1979).

2. Gjesdal (1976), Harris and Raviv (1976), (1979), Holmström (1977), (1979), Mirrlees (1976), Shavell

3. Ross $(1973 a, b)$, Spence and Zeckhauser (1971), Stiglitz (1974), Wilson (1968), (1969).

4. This is in contrast to the case of observable $X$ where choice of "ownership" is subsumed in the choice of sharing rule.

5. An interesting interpretation of $a$ for which the diagonality assumption holds, is the following: Assume the agent is allowed to receive some private information $\left(W: \Theta \rightarrow\left\{w_{i}\right\}\right)$ after contracting but before he makes his decision. Then $a$ may be interpreted as the agent's decision function (or strategy) with $a_{i}$ the choice made 
in response to the signal $w_{i}$. It follows that $a_{i}$ influences the random variables $X, Y, Z$ on the $\operatorname{set}\left\{\theta: W(\theta)=w_{i}\right\}$ only, and the matrix of derivatives of second-order is diagonal when it exists. In this model the objective is to induce the agent to use his private information Pareto efficiently. Hence it is an adverse selection problem formulated as a moral hazard problem.

6. Ross $(1973 a, b)$ derives these results as well. The reason why stronger conclusions cannot be obtained, is that the agent's action is undetermined when the sharing rule is constant ( $Y^{0}$ is employed). However, with a constant sharing rule, there is no risk-sharing and whatever action is chosen, $Y^{0}$ is inferior to perfect information i.e. the information system $(X, A)$.

7. Two other papers should be mentioned as well. Spence and Zeckhauser (1971) assume a special outcome structure and observation of $\theta, a$, and $x$ in various combinations. Townsend (1979) discusses risk-sharing when observation of $X$ is costly, but no incentive problem exists.

8. The converse is not true: $a^{*}$ may be inefficient given $S$, and (9) does not hold. This will be the case when $a^{*}$ is a minimum of the principal's evaluation measure with the second derivative sufficiently large. If the definition of the incentive problem is broadened to include these cases, Proposition 1 below will no longer hold. Holmström (1979) and Shavell (1979) impose restrictions on outcome functions to avoid this problem.

9. Gjesdal (1978) argues that it is possible to define a reasonable measure on the space of information systems, with the property that the set of information systems which satisfy (12) as well as (13) and/or (14) has measure zero. Note also that (12) is necessary but not sufficient for $Z$ to have no marginal value.

10. A slightly more general problem is to choose between $Z$ and $Y$ given a third information system $Y^{\prime}$. However, this reduces to a comparison of $\left(Z, Y^{\prime}\right)$ and $\left(Y, Y^{\prime}\right)$.

11. $\mathscr{E}$ may be referred to as the $\mathscr{A}$-relevant partition (Marschak and Miyasawa (1968)). Rather than assuming $\mathscr{A}$ finite, $X, Y$ and $Z$ might alternatively have been assumed measurable with respect to some finite partition of $\mathscr{A}$. Gjesdal (1978) has attempted to generalize some of the results of this section to more general action space and outcome functions.

12. Such a decision problem is not part of the agency problem. However, the agency problem is easily generalized by introducing a decision to be made (collectively or by the principal) after the signal $y$ is received. Then $Y$ is valuable for incentive-insurance as well as decision making purposes (see Gjesdal (1978)). Note that Condition $\mathrm{B}$, as defined here, ranks information systems for a fixed value of $a$.

13. Consider two modifications to the contract which pays the agent $E\left(S^{\prime} \mid y_{i}\right)$ when $y_{i}$ is observed. One modification pays $S^{\prime}(z)$ and amounts to randomizing the sharing rule $E\left(S^{\prime} \mid Y\right)$. The other modification deducts an amount $E\left(S^{\prime} \mid y_{i}\right)-S\left(y_{i}\right)$ for each signal $y_{i}$. The latter modification which in this sense employs a deductible, is Pareto-superior.

14. It is true that if the ranking which is being constructed shall hold for any measure on $\Theta$, then the counterexample require that infinite penalties be available. If $a_{1}$ is to be enforced with information system $Y$, the necessary penalty must approach $-\infty$ in utility units, as the probability of $e_{1}$ approaches zero.

15. If infinite penalties are not available, this probability will have a strictly positive lower bound. An interesting example of less information (according to the ranking by Condition BA) being as valuable as more, is the case of conditional investigations. (Demski and Feltham (1978), Baiman and Demski (1980a,b), Townsend (1979)). Rather than buying the information system $Z$, the information system $Y-\{$ buy $Z$ with probability $p$, use $Y^{0}$ otherwise - is chosen. For incentives $Y$ is as good as $Z$ (and it is presumably cheaper). It should be kept in mind, though, that for $Y$ to be as valuable as $Z$ for all agency problems, $Y$ should also enable the agency to share risk equally well.

16. (11) implies Condition A. The converse is not true. When (11) holds, it is also true that $Y$ is ranked as high as $(Y, Z)$ by Condition $\mathrm{A}$. Hence if there is no risk sharing problem, $Y$ is as valuable as $(Y, Z)$, and $Z$ will not have marginal value given $Y$. Holmström ([1979], Proposition 3-necessity) proves this result for $Y=X$. His result is essentially a special case of Corollary 1 as observable $X$ is another case of no risk-sharing problem. In general Proposition 2 may be used to prove converses of Proposition 1.

17. Grossman and Hart (1981) also prove the sufficiency of Condition BA assuming the agent's utility function is of the form:

$$
V(s, a)=V_{1}(s) V_{2}(a)+V_{3}(a) .
$$

The additive form (as well as the multiplicative form) is a special case of this. With reference to the discussion of randomization below, it is interesting to note that this is precisely the class of utility functions for which risk preferences do not depend on action choice.

18. Weiss (1976) shows that when using a linear income tax, it may be optimal to make the tax rate random. The linear income tax model is essentially an agency problem with linear sharing rule. However, it is the imposed linearity which accounts for the efficiency of randomization in Weiss' case.

19. The likelihood matrix of $Y^{0}$ is a column vector of 1 's. That of a randomization is a matrix with identical rows. The Markov matrix is the row vector which is equal to the rows of this likelihood matrix. The relationship between imperfect information and randomization is interesting: Explicit randomizations are seldom seen, whereas imperfect information is used all the time.

20. Gjesdal (1978) provides a general analysis of this case.

21. Formally, the marginal salary increase considered may be expressed as $(d h) T$ where $T$ is a random variable with mean 1 . 


\section{REFERENCES}

BAIMAN, S. and DEMSKI, J. (1980a), "Variance Analysis Procedures as Motivational Devices", Management Science.

BAIMAN, S. and DEMSKI, J. (1980 b), "Economically Optimal Performance Evaluation and Control Systems" (Paper presented at the 15th Annual Conference in Accounting, University of Chicago).

BLACKWELL, F. (1951), "Comparisons of Experiments", in Neyman, J. (ed.) Proceedings of the Second Berkeley Symposium in Mathematical Statistics and Probability (University of California Press).

DEMSKI, J. and FELTHAM, G. (1978), "Economic Incentives in Budgetary Control Systems", Accounting Review.

GJESDAL, F. (1978), "Stewardship Accounting: Controlling Informational Externalities" (Unpublished Ph.D., Graduate School of Business, Stanford University).

GJESDAL, F. (1976), "Accounting in Agencies" (mimeo, Graduate School of Business, Stanford University).

GROSSMAN, S. J. and HART, O. D. (1981), "An Analysis of the Principal-Agent Problem" (mimeo).

HARRIS, M. and RAVIV, A. (1976), "Optimal Incentive Contracts with Imperfect Information" (Working Paper No. 70-75-76, Graduate School of Industrial Administration, Carnegie-Mellon University).

HARRIS, M. and RAVIV, A. (1979), "Optimal Contracts with Imperfect Information", Journal of Economic Theory.

HOLMSTRÖM, B. (1979), "Moral Hazard and Observability”, Bell Journal of Economics.

HOLMSTRÖM, B. (1977), "On Incentives and Control in Organizations" (Unpublished Ph.D., Grad. School of Business, Stanford University).

HORNGREN, C. (1977), "Cost Accounting: A Managerial Emphasis" (New Jersey).

MARSCHAK, J. and MIYASAWA, K. (1968), "Economic Comparability of Information Systems", Interna tional Economic Review.

MIRRLEES, J. A. (1975), "The Theory of Moral Hazard and Unobservable Behaviour" (Mimeo, Oxford).

MIRRLEES, J. A. (1976), "The Optimal Structure of Incentives and Authority within an Organization", Bell Journal of Economics.

ROSS, S. (1973a), "On the Economic Theory of Agency: The Principal's Problem", American Economic Review.

ROSS, S. (1973b), "The Economic Theory of Agency: The Principle of Similarity" (Proceedings of the NBER-NSF Conference on Decision Making and Uncertainty).

SHAVELL, S. (1979), "Risk Sharing and Incentives in the Principal and Agent Relationship", Bell Journal of Economics.

SPENCE, A. M. and ZECKHAUSER, R. (1971), "Insurance, Information and Individual Action", American Economic Review.

STIGLITZ, J. E. (1974), "Incentives and Risk Sharing in Sharecropping", Review of Economic Studies.

TOWNSEND, R. M. (1979), "Optimal Contracts and Competitive Markets with Costly State Verification", Journal of Economic Theory, 21.

WEISS, L. (1976), "The Desireability of Cheating Incentives and Randomness in the Optimal Income Tax", Journal of Political Economy.

WILSON, R. (1968), "On the Theory of Syndicates", Econometrica.

WILSON, R. (1969), "The Structure of Incentives for Decentralization under Uncertainty", La Decision. 